\title{
Optimization of Lathe Cutting Parameters Using Taguchi Method and Grey Relational Analysis
}

\author{
Bo-Lin Jian, ${ }^{1}$ Cheng-Chi Wang, ${ }^{2}$ Her-Terng Yau, ${ }^{1}$ \\ $\mathrm{Li}$-Wei $\mathrm{Wu},{ }^{1}$ and An-Hong Tian ${ }^{3 *}$ \\ ${ }^{1}$ Department of Electrical Engineering, National Chin-Yi University of Technology, \\ Taichung 41170, Taiwan \\ ${ }^{2}$ Graduate Institute of Precision Manufacturing, National Chin-Yi University of Technology, \\ Taichung 41170, Taiwan \\ ${ }^{3}$ College of Information Engineering, Qujing Normal University, Qujing 655011, China
}

(Received February 8, 2019; accepted December 27, 2019)

Keywords: Taguchi method, lathe, optimization, grey relational analysis

In the current precision industry, the rapid production of high-quality parts in bulk quantities has led to high competitiveness. In this study, the Taguchi method and grey relational analysis (GRA) approach were used in a practical investigation of precision lathe processing. The purpose was to find optimal parameters for single-target and multitarget cutting. The production of targets of the highest quality was the research focus, with the aim of strengthening the links between this study and the application to the processing industry. Precision, surface roughness, and material removal rate were selected as targets for improvement. The parameters commonly used for lathe processing were set as control factors, and cutting depth, spindle speed, feed rate, and material elongation were set as experimental factors. The results showed that in the cutting of materials, cutting precision was mainly affected by the depth of cut and spindle speed, surface roughness by spindle speed, and the material removal rate by the cutting depth. In a comparison of the quality loss for the same materials using previous parameters, the cutting precision has about 64 to $99 \%$ optimization, the surface roughness has 69 to $96 \%$ optimization, and the material removal rate has more than $90 \%$ optimization. GRA was also employed to analyze the sequences of parameters from the Taguchi experiments to obtain the target relationships and to find the various combinations of factors for improvement.

\section{Introduction}

The machine tool industry occupies an indispensable position in the development of a country. The precision of processing is continuously increasing in the pursuit of rapid mass production and the quality of products. These aspects of production have become very important and many studies have been conducted to search for ways in which the efficiency and quality of production can be improved. Controllable conditions that have an effect on the quality of the machine turning or milling process include the spindle speed, feed rate, cutting

*Corresponding author: e-mail: tianah@mail.qjnu.edu.cn https://doi.org/10.18494/SAM.2020.2674 
fluid, cutting depth, shank angle, and the lathe tools or milling cutters used. Many studies on all these aspects have been conducted, ${ }^{(1,2)}$ particularly on cutting tool wear. ${ }^{(3-6)}$ Bharilya et al. ${ }^{(7)}$ used a dynamometer and other devices to investigate the parameters that might reduce cutting force and increase cutting speed. Pislaru et al. ${ }^{(8)}$ used wavelet transformation to identify the resonance frequencies in machine tools and the machine status. Rmili et al. ${ }^{(9)}$ used an acceleration gauge to obtain wear vibration characteristics. This was done to determine whether average power signal processing analysis can be used to develop an automatic detection system for the analysis of tool wear. Cuka and $\mathrm{Kim}^{(10)}$ used a dynamometer (a microphone and an accelerometer) to collect signals during cutting and fuzzy clustering to analyze the cutting tool status. To establish the values of parameters in the above research, the costs of data collection and sensors were high, and the material cost of many repeated experiments was also a considerable burden.

The Taguchi method was used in this study to determine the required number of machine experiments and to collect data that can be used for effective analysis. The desired quality characteristics and the experimental factors that may affect quality were selected as reference standards. The standards for a specific item were set using the best combinations that could be found. Pang et al. ${ }^{(11)}$ used the Taguchi method to optimize processing parameters for specific materials. Das et al. ${ }^{(12)}$ used the grey fuzzy algorithm and Taguchi method to find the best parameters for cutting $\mathrm{Al}-4.5 \% \mathrm{Cu}-\mathrm{TiC}$ metal composite material on milling machines. Das et al. ${ }^{(13)}$ used fuzzy theory coupled with the Taguchi method to optimize material parameters. Gupta et al. ${ }^{(14)}$ also used the Taguchi and fuzzy methods to study parameter optimization using AISI P-20 steel. Asiltürk et al. ${ }^{(15)}$ used the Taguchi quality method and response surface methodology (RSM) to conduct research on surface roughness using the medical material Co28Cr6Mo. Li et al. ${ }^{(16)}$ also used parameter correlation, RSM, and multiobjective swarm optimization (MOPSO) in the Taguchi method to assist in a search for optimal production efficiency. Ajith Arul Daniel et al. ${ }^{(17)}$ used an artificial neural network (ANN) to carry out prediction and parameter optimization research on Taguchi quality and grey relational analysis (GRA) to examine milling machine performance. Thankachan et al. ${ }^{(18)}$ used the Taguchi method, GRA, and an ANN to predict and optimize the surface roughness of products made of aluminum alloys and the material removal rate. Tamiloli et al. ${ }^{(19)}$ performed GRA using Taguchi factorial experiments and developed an adaptive neuro-fuzzy inference system (ANFIS) model to optimize parameter selection. This showed that GRA can use the signal-to-noise ratio $(\mathrm{S} / \mathrm{N})$ obtained from Taguchi experiments for relational analysis. The primary use of the Taguchi method is to reduce the number of experiments, and grey relations produce good results for the analysis of data from short sequences.

However, all these previous studies involved the use of a single material or tool to find the best parameters. Here, we look at the optimization parameters for different materials by examining different common materials processed by the same machine. Workpiece precision, which was not previously a research priority, was a goal in this study and workpiece surface roughness and material removal rate (MRR) were focused on. Ceramic tools, now commonly used in industry, were used in turning experiments with various metals. 


\section{Methods}

To implement the optimized parameters in lathe processing, the Taguchi method and GRA theory were both used, and the experimental flow is shown in Fig. 1.

To explain the framework of the experimental process in detail, experiment-related devices are introduced in Sect. 2.1, and the Taguchi theory and GRA are introduced in Sect. 2.2.

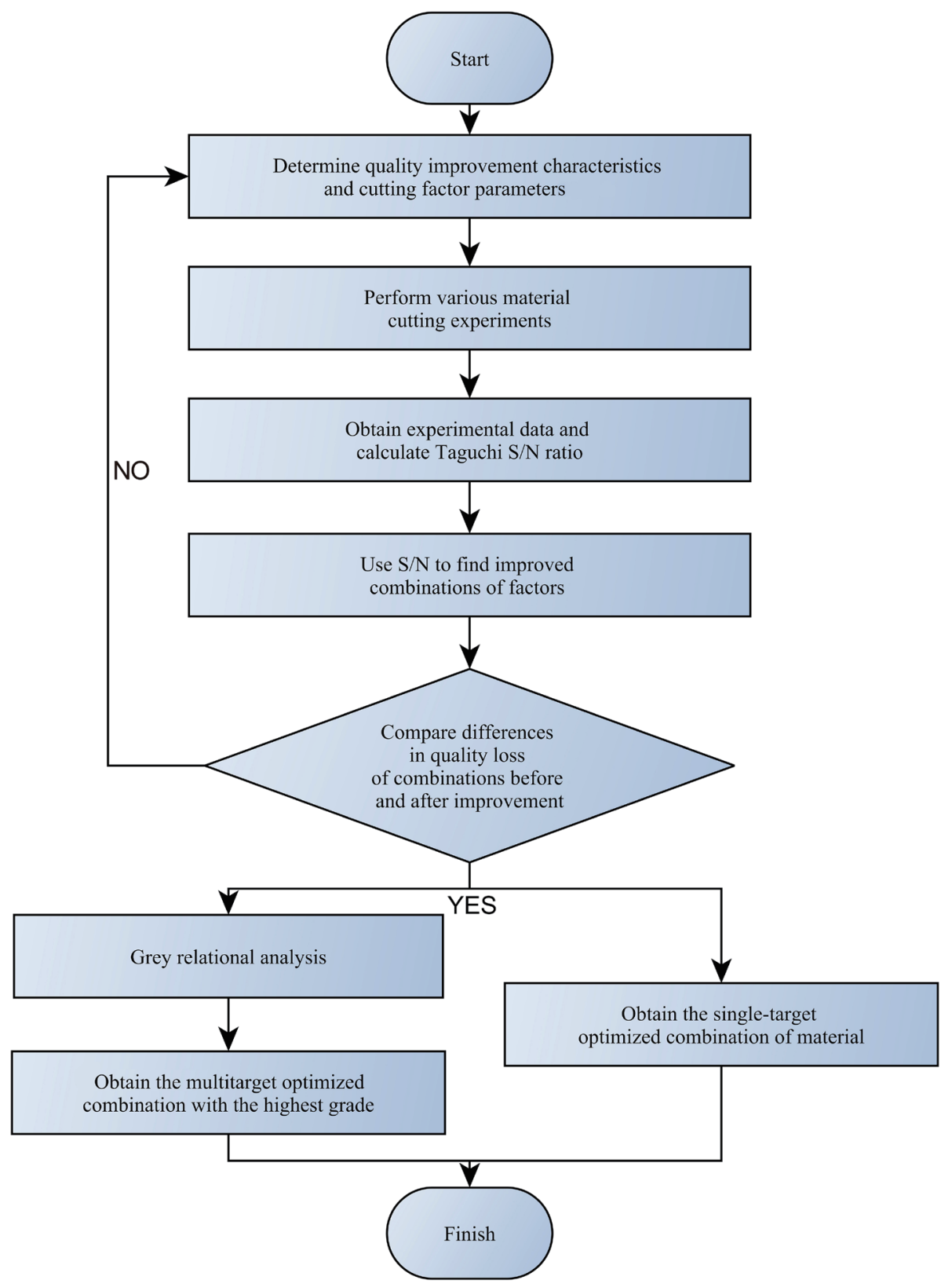

Fig. 1. (Color online) Flowchart of the experiment. 


\subsection{Specifications of experimental equipment}

The machine used was a Mike Machine spherical digital computer numerical control (CNC) (Model MC4200BL), as shown in Fig. 2. The machine had a POSA TAC-10-CY spindle and a SYNTEC 21-TA controller.

The cutting tools used in this study were ceramic and the materials used in the experiments were S45C medium-carbon, S20C low-carbon, SCM415 chromium alloy, 304 stainless, and SCM440 nickel steels, all commonly used in industry. See Table 1 for detailed specifications.

CHAIN ETQNL-2020K16 cutting tools were used. To measure the surface roughness of workpieces, a Mitutoyo SJ-210 surface roughness meter was used. A Carl Zeiss Contura was also used for roughness measurements.

\subsection{Taguchi theory and GRA}

To reduce the number of experiments needed to find optimal parameters and to make it easy for machine operators to rapidly select them, the Taguchi method ${ }^{(20)}$ is used. Genichi Taguchi, in his original work, combined money and loss expressed by quality, not only taking cost into account, but also including consumer and social aspects to arrive at a "loss function". In

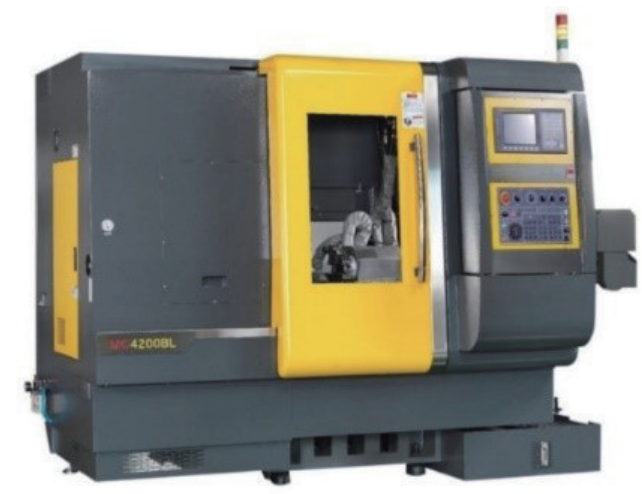

Fig. 2. (Color online) Spherical digital CNC.

Table 1

Workpiece composition in percentages.

\begin{tabular}{lccccc}
\hline \multirow{2}{*}{ Material } & \multicolumn{5}{c}{ Component } \\
\cline { 2 - 6 } & Medium-carbon steel & Low-carbon steel & Chromium alloy steel & Stainless steel & Nickel steel \\
\hline $\mathrm{C}$ & 0.460 & 0.160 & 0.13 & 0.059 & 0.38 \\
$\mathrm{P}$ & 0.013 & 0.015 & 0.03 & 0.032 & 0.03 \\
$\mathrm{Mn}$ & 0.790 & 0.410 & 0.60 & 1.82 & 0.60 \\
$\mathrm{~S}$ & 0.007 & 0.007 & 0.03 & 0.0283 & 0.03 \\
$\mathrm{Si}$ & 0.220 & 0.220 & 0.15 & 0.46 & 0.15 \\
$\mathrm{Ni}$ & 0.020 & 0.010 & 0.25 & 0.54 & 0.25 \\
$\mathrm{Cr}$ & 0.140 & 0.020 & 0.90 & 8.10 & 0.90 \\
$\mathrm{Cu}$ & 0.010 & $\mathrm{Tr}$ & - & 18.69 & 0.30 \\
$\mathrm{Mo}$ & - & - & 0.15 & 0.12 & 0.15 \\
\hline
\end{tabular}


product manufacture, the target value is contained in the objective. The purpose of quality is to achieve the target value, and to do this, product variation has to be reduced. Furthermore, to achieve a stable product quality, it is necessary to find the design value parameters. To change these parameters, one must start with the controllable factors involved in production and adjust them without incurring large costs. In the Taguchi method, an orthogonal array is used to rank parameter factor levels. Then, by the standard integration of the factors, the $\mathrm{S} / \mathrm{N}$ ratios of the combinations can be computed.

The loss function of Taguchi quality is a function of both quality and money. The loss function is expressed by a Tate series: ${ }^{(20,21)}$

$$
L(y)=K \times(y-m)^{2},
$$

where $m$ is the target value, $y$ is the characteristic value, and $K$ is the quality loss coefficient (the constant used in measurements).

The improvement targets have expected trends. The status of Taguchi quality can be the larger the better (LTB), the smaller the better (STB), or nominal the best (NTB). LTB is characterized by the largest actual expected value, STB is characterized by the smallest actual expected value, while NTB is between them and is characterized by "expecting the actual value to be close to the target value". In this study, LTB and STB were the experimental targets. As far as cutting error and surface roughness were concerned, the smaller the error and Ra value, the better the processed workpiece. For the material removal rate, the larger the value, the higher the cutting efficiency.

Owing to prior knowledge of the correlation between the processing control factors and goal achievement, GRA was employed to accomplish the relational analysis of the factors and goals. GRA ${ }^{(22)}$ is an important part of grey theory and is a measurement method for analyzing discrete sequences. Compared with traditional statistical regression, GRA uses a small amount of data and multifactor data to analyze sequence relationships. GRA involves sequence data normalization and can speed up analysis and enhance accuracy. In this study, surface roughness and precision are STB and surface removal rates are LTB. Normalized actions are expressed as follows:

STB:

$$
x_{i}^{*}(k)=\frac{\max x_{i}(k)-x_{i}(k)}{\max x_{i}(k)-\min x_{i}(k)},
$$

LTB:

$$
x_{i}^{*}(k)=\frac{x_{i}(k)-\min x_{i}(k)}{\max x_{i}(k)-\min x_{i}(k)} .
$$

$x_{i}(k)$ is the largest or smallest in the same quality sequence and current data calculation results. After normalization, the data undergoes sequential grey relational coefficient (GRC) computation. ${ }^{(19)}$ The GRC equation is 


$$
\xi_{i}(k)=\frac{\Delta_{\min }+\xi \Delta_{\max }}{\Delta_{i}+\xi \Delta_{\max }}
$$

Here, $\Delta_{i}$ is the difference between the absolute values of the standard sequence and the compared sequence in the same row $\Delta_{\min }$, and $\Delta_{\max }$ is the difference between the maximum and minimum values of the two standards and compared sequences. $\zeta$ is the distinguishing coefficient, the function of which is to compare the background object with the object to be tested. Adjusting $\zeta$ will only affect the numerical value, not the sequence. Generally, $\zeta$ is adjusted to 0.5 , but this can be changed to suit actual needs.

Finally, the GRCs are sorted to find the grey relational grade (GRG). The GRG is calculated using the mean of the GRCs, as follows:

$$
\Upsilon_{i}=\frac{1}{n} \sum_{k=1}^{n} \xi_{i}(k)
$$

where $n$ is the output volume in the same row. The computed GRG value was used to rate the sizes of the grades and find the corresponding combination of factor parameters.

The $\mathrm{S} / \mathrm{N}$ obtained through the Taguchi quality method was computed to obtain the optimal parameters of single targets. The sequence analysis in GRA was then used to analyze Taguchi $\mathrm{S} / \mathrm{N}$ data and perform relational analysis to obtain multiple targets while improving the parameters.

\section{Experimental Results and Discussion}

The cutting precision, surface roughness, and MRRS were the quality characteristics in these experiments. Precision is a very important indicator of quality and $\mathrm{CNC}$ processing is a large, well-established industrial process. The error reduction leads to a great improvement in product yield rate and reduced costs and is the main reason for this in-depth study of lathe precision. Surface roughness is also a major product indicator and uneven surfaces will affect appearance as well as cause problems between moving contact surfaces. A rough surface will also increase wear, also an integral factor examined in this study. The MRR was calculated using the mass of the workpiece obtained before processing, as described by Shrivastava and Singh. ${ }^{(23)}$

To explain the experimental process framework in detail, the Taguchi experimental process is introduced in Sect. 3.1. Section 3.2 covers the GRA.

\subsection{Experimentation using the Taguchi method}

With reference to the literature, ${ }^{(1,8)}$ we varied the cutting depth, spindle speed, feed rate, and material elongation to establish the orthogonal array experimentally. Refer to Table 2 for a comparison of the control factor standards. 
Table 2

Control factor standards.

\begin{tabular}{|c|c|c|c|}
\hline \multirow{2}{*}{ Factor } & \multicolumn{3}{|c|}{ Standard } \\
\hline & 1 & 2 & 3 \\
\hline Cutting depth (mm) & 0.1 & 0.6 & 1.1 \\
\hline Spindle speed (rpm) & 2101 & 2334 & 2567 \\
\hline Feed rate (rev) & 0.15 & 0.2 & 0.25 \\
\hline Elongation (mm) & 40 & 60 & 80 \\
\hline
\end{tabular}

Table 3

Experimental data-medium-carbon and low-carbon steel quality characteristics.

\begin{tabular}{llllllll}
\hline & \multicolumn{3}{c}{ Medium-carbon steel } & & \multicolumn{3}{c}{ Low-carbon steel } \\
\cline { 2 - 3 } \cline { 6 - 7 } & $\begin{array}{c}\text { Precision } \\
\text { error }\end{array}$ & $\begin{array}{c}\text { Surface } \\
\text { roughness }\end{array}$ & \multicolumn{1}{c}{ MRR } & & $\begin{array}{c}\text { Precision } \\
\text { error }\end{array}$ & $\begin{array}{c}\text { Surface } \\
\text { roughness }\end{array}$ & \multicolumn{1}{c}{ MRR } \\
\hline 1 & 0.013617 & 1.8886667 & 0.090909091 & & 0.0085172 & 1.9026667 & 0.136363636 \\
2 & 0.0308 & 3.251667 & 0.125 & & 0.00513 & 3.464 & 0.222222222 \\
3 & 0.02955 & 4.947 & 0.071428571 & & 0.00493 & 5.045333 & 0.214285714 \\
4 & 0.03207 & 3.338 & 0.666666667 & & 0.01036 & 3.568 & 0.777777778 \\
5 & 0.0419 & 4.979333 & 0.8125 & & 0.01834 & 5.105667 & 0.8125 \\
6 & 0.01425 & 1.908 & 0.65 & & 0.006532 & 1.915 & 0.7 \\
7 & 0.00961 & 1.698333 & 1.375 & & 0.00524 & 5.085333 & 1.5625 \\
8 & 0.0053 & 1.951667 & 1.15 & & 0.02352 & 2.031333 & 1.25 \\
9 & 0.01391 & 3.409 & 1.277777778 & 0.005562 & 3.649667 & 1.333333333 \\
\hline
\end{tabular}

Table 4

Experimental data-chromium alloy, stainless, and nickel steel quality characteristics.

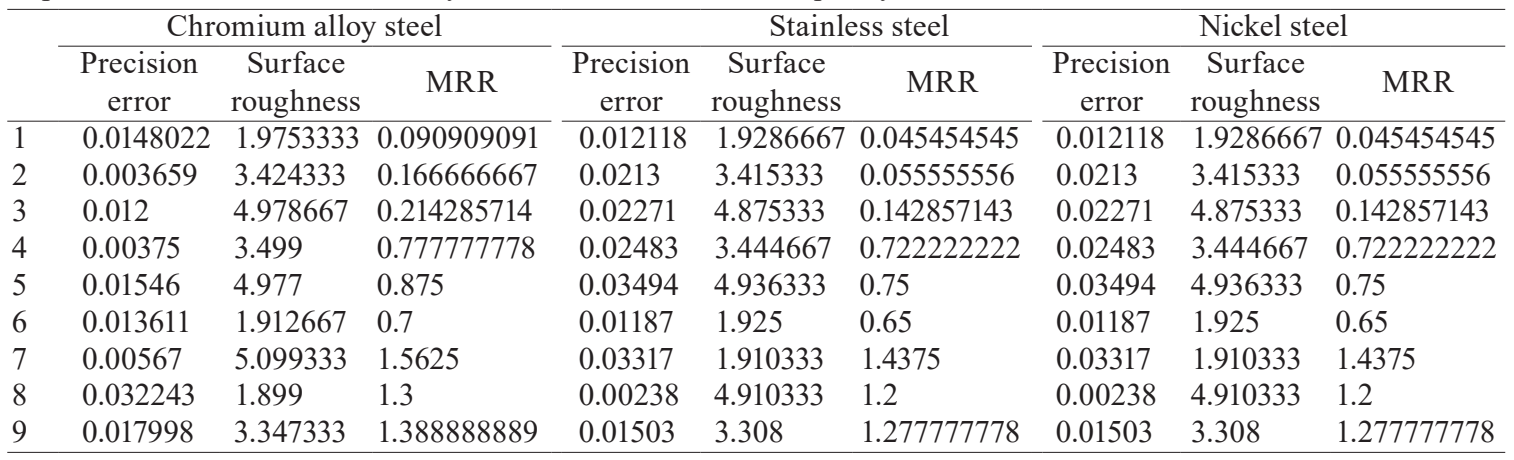

The orthogonal array selected in this study was $L_{9}\left(3^{4}\right)$. Nine experiments in total were performed and cutting was carried out in straight lines. The total cutting depth for each experiment was $30 \mathrm{~mm}$.

Table 3 shows the Taguchi orthogonal experimental data for medium-carbon and low-carbon steels. Table 4 shows the Taguchi orthogonal experimental data for chromium alloy, stainless, and nickel steels. The units of precision error, surface roughness, and MRR are $\mathrm{mm}, \mu \mathrm{m}$, and $\mathrm{g} / \mathrm{s}$, respectively.

According to the data measured from the respective targets, LTB and STB S/N ratios are substituted. The data of the materials obtained after computation are shown in Tables 5 and 6 .

On the basis of the computed data above, the tables of the target factors of the materials were established. Refer to Tables 7 and 8 for the cutting precision. 
Table 5

$\mathrm{S} / \mathrm{N}$ ratio-medium-carbon and low-carbon steel quality characteristics.

\begin{tabular}{|c|c|c|c|c|c|c|}
\hline & & Medium-carbon stee & & & Low-carbon & teel \\
\hline & Precision error & Surface roughness & MRR & Precision error & Surface roughness & MRR \\
\hline & $\mathrm{S} / \mathrm{N}$ ratio & $\mathrm{S} / \mathrm{N}$ ratio & $\mathrm{S} / \mathrm{N}$ ratio & $\mathrm{S} / \mathrm{N}$ ratio & $\mathrm{S} / \mathrm{N}$ ratio & $\mathrm{S} / \mathrm{N}$ ratio \\
\hline 1 & 37.318478 & -5.523106 & -20.8278537 & 41.394063 & -5.587254 & -17.3060285 \\
\hline 2 & 30.22868 & -10.2421 & -18.0617997 & 45.80166 & -10.7916 & -13.0642502 \\
\hline 3 & 30.59003 & -13.8868 & -22.9225607 & 46.14394 & -14.0578 & -13.3801356 \\
\hline 4 & 29.8778 & -10.4697 & -3.52182518 & 39.6894 & -11.0485 & -2.18288938 \\
\hline 5 & 27.55548 & -13.9434 & -1.80353260 & 34.73072 & -14.161 & -1.80353260 \\
\hline 6 & 36.92665 & -5.61157 & -3.74173286 & 43.69956 & -5.64338 & -3.0980392 \\
\hline 7 & 40.34466 & -4.60046 & 2.766053963 & 45.62001 & -14.1264 & 3.87640052 \\
\hline 8 & 45.5153 & -5.80811 & 1.213956807 & 32.57124 & -6.15562 & 1.93820026 \\
\hline 9 & 37.13383 & -10.6525 & 2.129106618 & 45.09538 & -11.2451 & 2.498774732 \\
\hline
\end{tabular}

Table 6

$\mathrm{S} / \mathrm{N}$ ratio-chromium alloy, stainless, and nickel steel quality characteristics.

\begin{tabular}{|c|c|c|c|c|c|c|c|c|c|}
\hline & \multicolumn{3}{|c|}{ Chromium alloy steel } & \multicolumn{3}{|c|}{ Stainless steel } & \multicolumn{3}{|c|}{ Nickel steel } \\
\hline & $\begin{array}{l}\text { Precision } \\
\text { error } \\
\mathrm{S} / \mathrm{N} \text { ratio }\end{array}$ & $\begin{array}{c}\text { Surface } \\
\text { roughness } \\
\mathrm{S} / \mathrm{N} \text { ratio }\end{array}$ & $\begin{array}{c}\text { MRR } \\
\text { S/N ratio }\end{array}$ & $\begin{array}{c}\text { Precision } \\
\text { error } \\
\mathrm{S} / \mathrm{N} \text { ratio }\end{array}$ & $\begin{array}{c}\text { Surface } \\
\text { roughness } \\
\mathrm{S} / \mathrm{N} \text { ratio }\end{array}$ & $\begin{array}{c}\text { MRR } \\
\text { S/N ratio }\end{array}$ & $\begin{array}{c}\text { Precision } \\
\text { error } \\
\text { S/N ratio }\end{array}$ & $\begin{array}{c}\text { Surface } \\
\text { roughness } \\
\mathrm{S} / \mathrm{N} \text { ratio }\end{array}$ & $\begin{array}{c}\text { MRR } \\
\mathrm{S} / \mathrm{N} \text { ratio }\end{array}$ \\
\hline 1 & 36.593484 & -5.912808 & -20.8278537 & 38.331118 & -5.705143 & -26.8484536 & 26.88675818 & -6.16132330 & -14.8072537 \\
\hline 2 & 48.73319 & -10.69 & -15.5630 & & -10.6687 & -25.1 & 61 & -11.5 & \\
\hline & 38.41 & -13.9 & -13 & & -13.7601 & -16 & 49 & -14.2 & \\
\hline & 48.5 & -10.8 & -2.18 & & -10.7429 & -2.8 & 91 & -11.2 & $-1 .($ \\
\hline 5 & 36.2156 & -13.93 & -1.15983894 & & -13.8681 & -2.49877473 & 34.550 & -14.3534100 & -0.56 \\
\hline & 37.32252 & -5.63279 & -3.0980392 & 38.51394 & -5.68861 & -3.74173286 & 26.40484233 & -6.13278882 & -1.93820026 \\
\hline 7 & 44.92834 & -14.1503 & 3.87640052 & 29.5 & -5.62218 & 3.15215 & 27.57732637 & -14.3976694 & 7563 \\
\hline 8 & 29.83 & -5.57 & 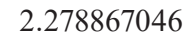 & 52 & -13.8222 & 1.58 & 23.1290701 & -6.64 & 2.60 \\
\hline & 34.89566 & -10.494 & 2.853350071 & 36.46074 & -10.3913 & 2.129106618 & 26.40941923 & -11.5467716 & 3.521825181 \\
\hline
\end{tabular}

Table 7

Factor results-medium-carbon and low-carbon steel cutting errors.

\begin{tabular}{|c|c|c|c|c|c|c|c|c|}
\hline \multirow{3}{*}{ Standard } & \multicolumn{8}{|c|}{ Factor } \\
\hline & \multicolumn{4}{|c|}{ Medium-carbon steel } & \multicolumn{4}{|c|}{ Low-carbon steel } \\
\hline & A & B & $\mathrm{C}$ & $\mathrm{D}$ & $\mathrm{A}$ & B & $\mathrm{C}$ & $\mathrm{D}$ \\
\hline 1 & 32.71239619 & 35.84698009 & 39.92014298 & 34.00259808 & 44.44655548 & 42.23448879 & 39.22162283 & 40.40672078 \\
\hline 2 & 31.45331268 & 34.43315718 & 32.41344036 & 35.83333092 & 39.37322635 & 37.70120702 & 43.52881237 & 45.04041069 \\
\hline 3 & 40.99793091 & 34.8835025 & 32.83005644 & 35.32771078 & 41.09554296 & 44.97962899 & 42.16488959 & 39.46819332 \\
\hline Difference & 9.544618227 & 1.413822912 & 7.506702624 & 1.830732838 & 5.073329123 & 7.278421967 & 4.307189537 & 5.572217367 \\
\hline
\end{tabular}

Table 8

Factor results-chromium alloy, stainless, and nickel steel cutting errors.

\begin{tabular}{|c|c|c|c|c|c|c|c|c|}
\hline \multirow{3}{*}{ Standard } & \multicolumn{8}{|c|}{ Factor } \\
\hline & \multicolumn{4}{|c|}{ Chromium alloy steel } & \multicolumn{4}{|c|}{ Stainless steel } \\
\hline & A & $\mathrm{B}$ & $\mathrm{C}$ & $\mathrm{D}$ & A & $\mathrm{B}$ & $\mathrm{C}$ & $\mathrm{D}$ \\
\hline 1 & 41.24754948 & 43.3507099 & 34.58240139 & 35.90158282 & 34.87879543 & 33.3390262 & 43.10852281 & 34.64187024 \\
\hline 2 & 40.68947635 & 38.25999752 & 44.0530511 & 43.66134808 & 33.24934134 & 38.34845045 & 33.99739007 & 33.84355002 \\
\hline 3 & 36.55173333 & 36.87805174 & 39.85330667 & 38.92582825 & 39.50895968 & 35.9496198 & 30.53118358 & 39.15167619 \\
\hline Difference & 4.695816146 & 6.472658155 & 9.470649712 & 7.759765263 & 6.259618343 & 5.009424247 & 12.57733923 & 5.30812617 \\
\hline
\end{tabular}

\begin{tabular}{llccc}
\hline \multirow{2}{*}{ Standard } & \multicolumn{4}{c}{ Factor } \\
\cline { 2 - 5 } & \multicolumn{4}{c}{ Nickel steel } \\
\cline { 2 - 5 } & \multicolumn{1}{c}{$\mathrm{A}$} & $\mathrm{B}$ & $\mathrm{C}$ & $\mathrm{D}$ \\
\hline 1 & 29.34528276 & 28.20955455 & 25.47355687 & 29.28217831 \\
2 & 30.37325964 & 29.08528207 & 28.71680565 & 27.85286244 \\
3 & 25.7052719 & 28.12897768 & 31.23345179 & 28.28877356 \\
Difference & 4.667987743 & 0.95630439 & 5.759894916 & 1.429315868 \\
\hline
\end{tabular}


The obtained factor values were imported into software and presented in resonance diagrams to identify the difference in $\mathrm{S} / \mathrm{N}$ ratio between various factor standards. Figure 3 shows cutting precision resonance diagrams.

Tables 9 and 10 are the tabulated factor results of surface roughness. Figure 4 shows surface roughness resonance diagrams.

Tables 11 and 12 are the factor results of MRRs. Figure 5 shows MRR resonance diagrams.
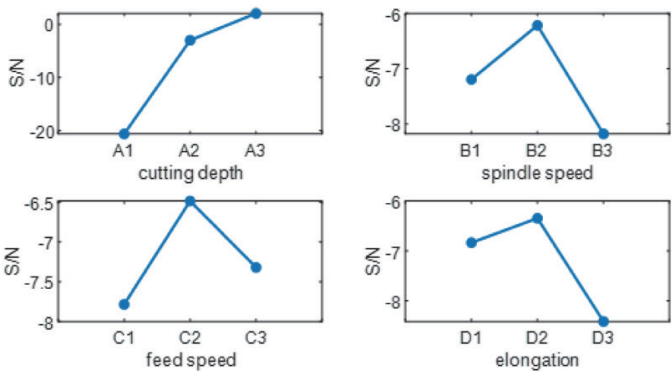

(a)
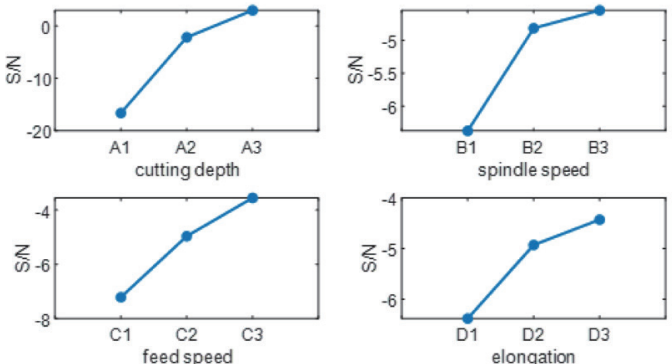

(c)
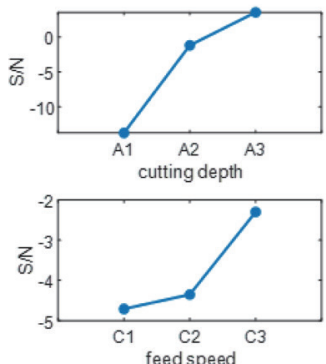
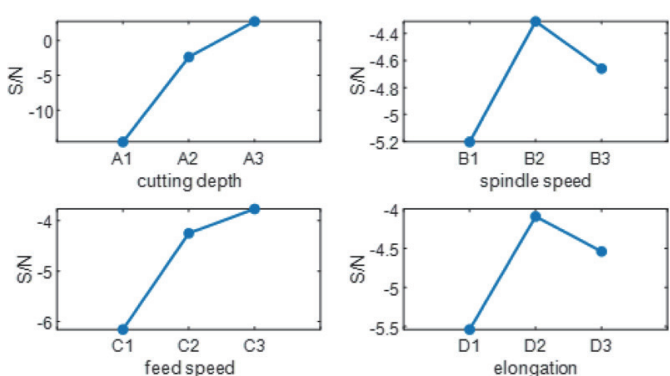

(b)
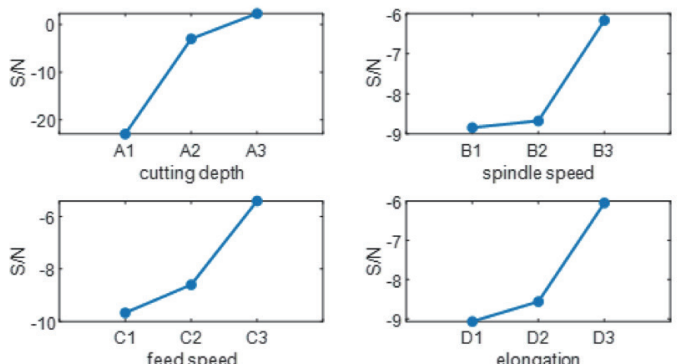

(d)
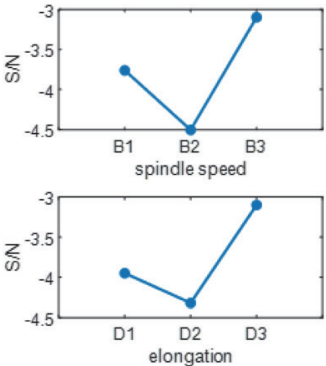

(e)

Fig. 3. (Color online) Resonance diagrams of cutting errors: (a) medium-carbon, (b) low-carbon, (c) chromium alloy, (d) stainless, and (e) nickel steels.

Table 9

Factor results-medium-carbon and low-carbon steel surface roughnesses.

\begin{tabular}{|c|c|c|c|c|c|c|c|c|}
\hline \multirow{3}{*}{ Standard } & \multicolumn{8}{|c|}{ Factor } \\
\hline & \multicolumn{4}{|c|}{ Medium-carbon steel } & \multicolumn{4}{|c|}{ Low-carbon steel } \\
\hline & A & B & $\mathrm{C}$ & $\mathrm{D}$ & A & B & $\mathrm{C}$ & $\mathrm{D}$ \\
\hline 1 & -9.884021632 & -6.864430543 & -5.647595537 & -10.03969011 & -10.14553638 & -10.25404654 & -5.795417888 & -10.33112246 \\
\hline 2 & -10.00823935 & -9.997885761 & -10.45479568 & -6.81804882 & -10.28430721 & -10.36941024 & -11.02837287 & -10.18710725 \\
\hline 3 & -7.02037053 & -10.05031521 & -10.8102403 & -10.05489258 & -10.50902548 & -10.31541229 & -14.11507832 & -10.42063937 \\
\hline Difference & 2.987868824 & 3.18588467 & 5.162644759 & 3.236843763 & 0.363489094 & 0.115363703 & 8.319660427 & 0.233532122 \\
\hline
\end{tabular}


Table 10

Factor results-chromium alloy, stainless, and nickel steel surface roughnesses.

\begin{tabular}{|c|c|c|c|c|c|c|c|c|}
\hline \multirow{3}{*}{ Standard } & \multicolumn{8}{|c|}{ Factor } \\
\hline & \multicolumn{4}{|c|}{ Chromium alloy steel } & \multicolumn{4}{|c|}{ Stainless steel } \\
\hline & A & $\mathrm{B}$ & $\mathrm{C}$ & $\mathrm{D}$ & A & $\mathrm{B}$ & $\mathrm{C}$ & $\mathrm{D}$ \\
\hline 1 & -10.18219651 & -10.31398491 & -5.705364309 & -10.11537997 & -10.04463057 & -7.356756872 & -8.405325888 & -9.988181026 \\
\hline 2 & -10.15033915 & -10.06712426 & -10.68812625 & -10.1581915 & -10.09988276 & -12.78632367 & -10.600972 & -7.326486563 \\
\hline 3 & -10.07158219 & -10.02300868 & -14.01062729 & -10.13054638 & -9.945237529 & -9.946670327 & -11.08345298 & -12.77508328 \\
\hline Difference & 0.110614314 & 0.290976234 & 8.305262977 & 0.042811526 & 0.154645236 & 5.429566795 & 2.678127089 & 5.448596715 \\
\hline
\end{tabular}

\begin{tabular}{lrrrr}
\hline \multirow{2}{*}{ Standard } & \multicolumn{4}{c}{ Factor } \\
\cline { 2 - 5 } & \multicolumn{4}{c}{ Nickel steel } \\
\hline 1 & -10.65616776 & -10.61270069 & -6.312946812 & -10.68716834 \\
2 & -10.58843606 & -10.86333329 & -11.47258082 & -10.70743993 \\
3 & -10.86305648 & -10.63162631 & -14.32213267 & -10.71305203 \\
Difference & 0.274620415 & 0.250632598 & 8.009185854 & 0.025883689 \\
\hline
\end{tabular}
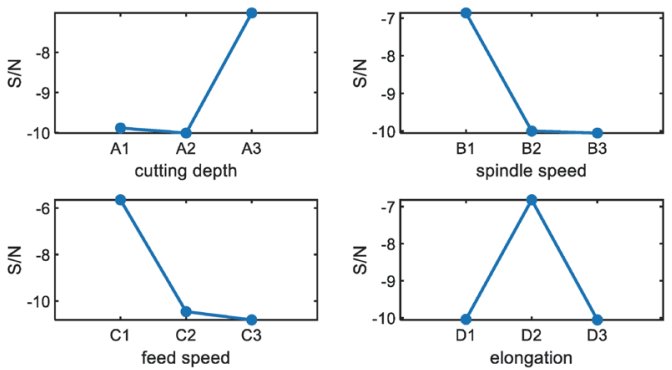

(a)
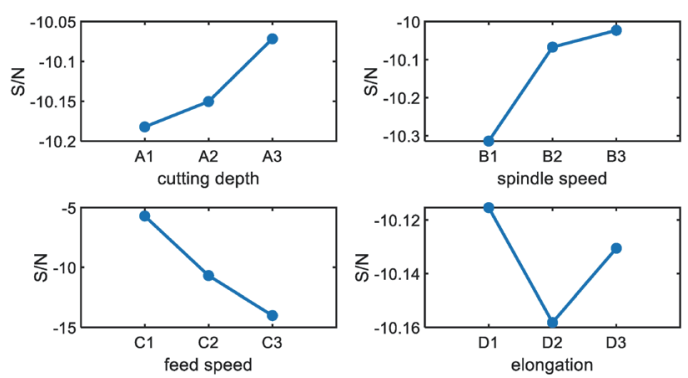

(c)
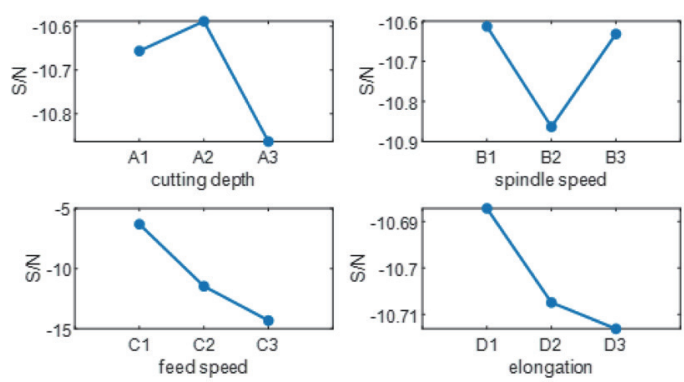

(e)
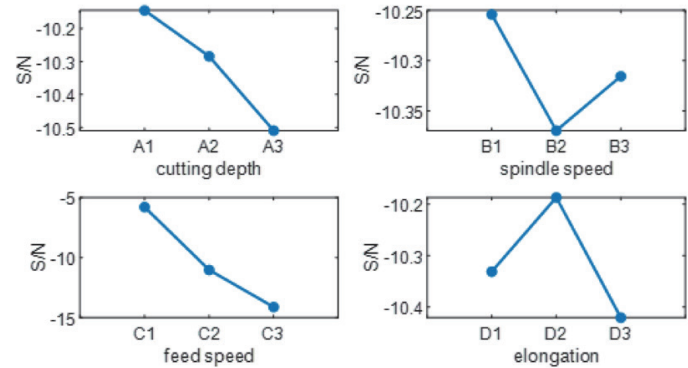

(b)
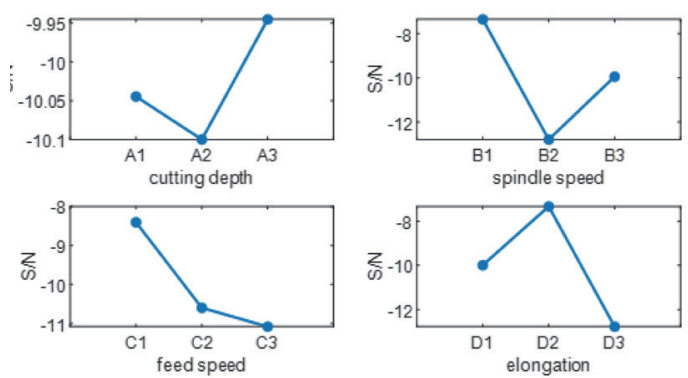

(d)

Fig. 4. (Color online) Resonance diagrams of surface roughness: (a) medium-carbon, (b) low-carbon, (c) chromium alloy, (d) stainless, and (e) nickel steels. 
Table 11

Factor results-medium-carbon and low-carbon steel MRRs.

\begin{tabular}{|c|c|c|c|c|c|c|c|c|}
\hline \multirow{3}{*}{ Standard } & \multicolumn{8}{|c|}{ Factor } \\
\hline & \multicolumn{4}{|c|}{ Medium-carbon steel } & \multicolumn{4}{|c|}{ Low-carbon steel } \\
\hline & A & $\mathrm{B}$ & $\mathrm{C}$ & $\mathrm{D}$ & A & $\mathrm{B}$ & $\mathrm{C}$ & $\mathrm{D}$ \\
\hline 1 & -20.60407139 & -7.19454164 & -7.785209921 & -6.834093231 & -14.58347147 & -5.204172463 & -6.155289154 & -5.536928799 \\
\hline 2 & -3.022363552 & -6.21712518 & -6.484839434 & -6.345826215 & -2.361487065 & -4.309860874 & -4.249454977 & -4.095296318 \\
\hline 3 & 2.036372463 & -8.178395654 & -7.320013119 & -8.410143029 & 2.771125171 & -4.659800029 & -3.769089235 & -4.541608249 \\
\hline Difference & 22.64044385 & 1.961270474 & 1.300370487 & 2.064316815 & 17.35459664 & 0.894311589 & 2.386199919 & 1.441632481 \\
\hline
\end{tabular}

Table 12

Factor results-chromium alloy, stainless, and nickel steel MRRs.

\begin{tabular}{|c|c|c|c|c|c|c|c|c|}
\hline \multirow{3}{*}{ Standard } & \multicolumn{8}{|c|}{ Factor } \\
\hline & \multicolumn{4}{|c|}{ Chromium alloy steel } & \multicolumn{4}{|c|}{ Stainless steel } \\
\hline & A & B & $\mathrm{C}$ & $\mathrm{D}$ & A & B & $\mathrm{C}$ & $\mathrm{D}$ \\
\hline 1 & -16.59033811 & -6.37811419 & -7.215675286 & -6.37811419 & -22.95195484 & -8.840959868 & -9.668853854 & -9.072707243 \\
\hline 2 & -2.146922509 & -4.814665634 & -4.964188108 & -4.928221229 & -3.022363552 & -8.673533304 & -8.600975513 & -8.565008634 \\
\hline 3 & 3.002872546 & -4.541608249 & -3.554524679 & -4.428052654 & 2.288296202 & -6.171529016 & -5.416192822 & -6.048306312 \\
\hline Difference & 19.59321066 & 1.836505941 & 3.661150606 & 1.950061537 & 25.24025104 & 2.669430852 & 4.252661032 & 3.024400932 \\
\hline
\end{tabular}

\begin{tabular}{lcccc}
\hline \multicolumn{5}{c}{ Factor } \\
\hline \multirow{2}{*}{ Standard } & \multicolumn{4}{c}{ Nickel steel } \\
\cline { 2 - 5 } & $\mathrm{A}$ & $\mathrm{B}$ & $\mathrm{C}$ & $\mathrm{D}$ \\
\hline 1 & -13.75054656 & -3.761809536 & -4.712926227 & -3.948667694 \\
2 & -1.173941727 & -4.50564137 & -4.354750092 & -4.318783213 \\
3 & 3.55779206 & -3.099245322 & -2.29901991 & -3.099245322 \\
Difference & 17.30833862 & 1.406396048 & 2.413906317 & 1.219537891 \\
\hline
\end{tabular}
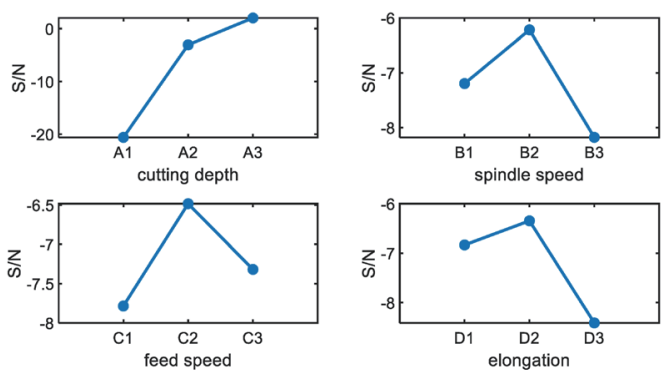

(a)
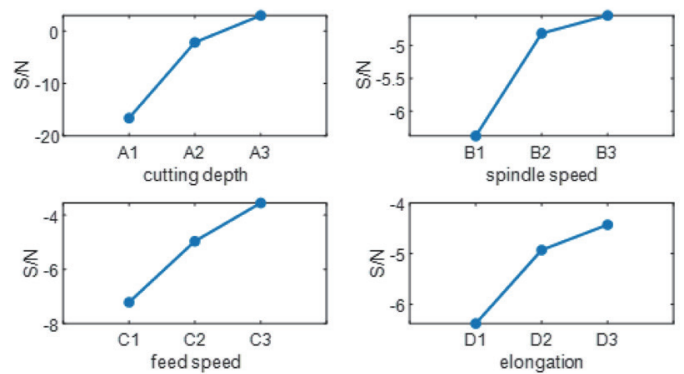

(c)
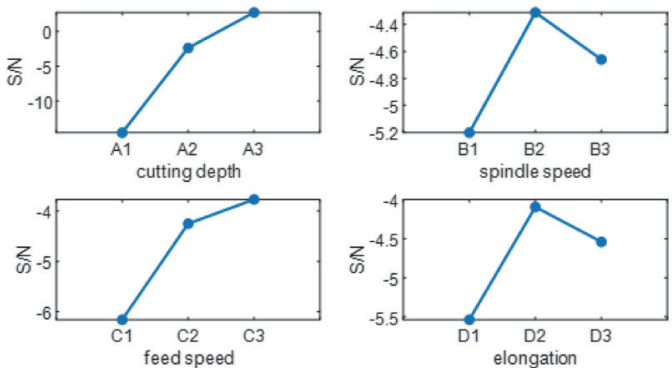

(b)
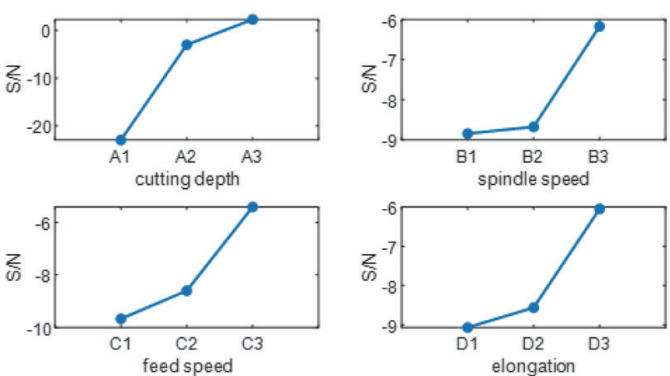

(d)

Fig. 5. (Color online) Resonance diagrams of MRRs: (a) medium-carbon, (b) low-carbon, (c) chromium alloy, and (d) stainless steels. 

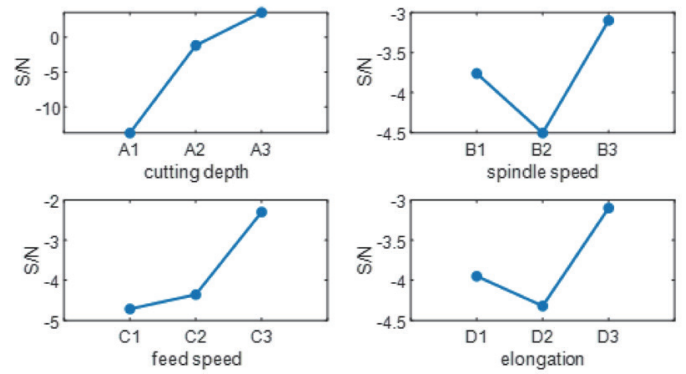

(e)

Fig. 5. (Color online) (Continued) Resonance diagrams of MRRs: (e) nickel steel.

Table 13

Best configuration combinations of material factors.

\begin{tabular}{lccccc}
\hline & $\begin{array}{c}\text { Medium-carbon } \\
\text { steel }\end{array}$ & $\begin{array}{c}\text { Low-carbon } \\
\text { steel }\end{array}$ & $\begin{array}{c}\text { Chromium alloy } \\
\text { steel }\end{array}$ & $\begin{array}{c}\text { Stainless } \\
\text { steel }\end{array}$ & $\begin{array}{c}\text { Nickel } \\
\text { steel }\end{array}$ \\
\hline \multirow{2}{*}{ Cutting precision } & A3B1 & A1B3 & A1B1 & A3B2 & A2B2 \\
& C1D2 & C2D2 & C2D2 & C1D3 & C3D1 \\
\hline \multirow{2}{*}{ Surface roughness } & A3B1 & A1B1 & A3B3 & A3B1 & A2B1 \\
& C1D2 & C1D2 & C1D1 & C1D2 & C1D1 \\
\hline \multirow{2}{*}{ Material removal rate } & A3B2 & A3B2 & A3B3 & A3B3 & A3B3 \\
& C2D2 & C3D2 & C3D3 & C3D3 & C3D3 \\
\hline
\end{tabular}

Table 14

Reduction percentages of quality loss for different material combinations.

\begin{tabular}{lccccc}
\hline & $\begin{array}{c}\text { Medium-carbon } \\
\text { steel (\%) }\end{array}$ & $\begin{array}{c}\text { Low-carbon } \\
\text { steel (\%) }\end{array}$ & $\begin{array}{c}\text { Chromium alloy } \\
\text { steel (\%) }\end{array}$ & $\begin{array}{c}\text { Stainless } \\
\text { steel (\%) }\end{array}$ & Nickel steel (\%) \\
\hline Cutting precision & 98.61 & 65.59 & 96.22 & 99.13 & 64.52 \\
Surface roughness & 96.10 & 71.41 & 69.04 & 82.40 & 70.12 \\
Material removal rate & 99.69 & 98.91 & 99.49 & 99.92 & 99.04 \\
\hline
\end{tabular}

The Taguchi quality calculation shows the impact of the factor standards of the materials from which the factor standard combinations most suitable for the targets were selected. We computed the quality loss of the originally set factor combinations and the improved best combinations to determine the amount of loss reduction. The previous experimental parameters and materials were set as $\mathrm{A} 1 \mathrm{~B} 3 \mathrm{C} 2 \mathrm{D} 1$, and the improved factor configuration is shown in Table 13.

The results of the computed quality loss are shown in Table 14. This table shows that the best combinations can reduce the quality loss of many preset combinations. The best parameters can be obtained to carry out parameter optimization on single targets.

\subsection{GRA}

Taguchi quality involves the computation of $\mathrm{S} / \mathrm{N}$ to determine which experimental combination of factors is optimal for single targets. Here, the sequence data under the Taguchi orthogonal experiment was used to perform GRA. The target quality underwent multitarget optimization. Tables 15 and 16 show the normalized $\mathrm{S} / \mathrm{N}$ ratios and respective target sequences 
obtained after GRC and GRG analyses. The material analysis results are shown in Tables 17 and 18.

Observations of the material grade show that a higher GRG produces a higher overall impact. It was found that the eighth combination is the best for medium-carbon steel, the sixth combination is best for low-carbon steel, the eighth combination is best for chromium alloy steel, the seventh combination is best for stainless steel, and the eighth combination is best for nickel steel. Thus, the eighth combination produces beneficial results for the optimization of various materials.

Table 15

Normalized S/N data-medium-carbon and low-carbon steel data.

\begin{tabular}{lllllllll}
\hline & \multicolumn{3}{c}{ Medium-carbon steel } & & \multicolumn{3}{c}{ Low-carbon steel } \\
\cline { 2 - 3 } \cline { 6 - 7 } & $\begin{array}{c}\text { Precision } \\
\text { error }\end{array}$ & $\begin{array}{c}\text { Surface } \\
\text { roughness }\end{array}$ & MRR & & $\begin{array}{c}\text { Precision } \\
\text { error }\end{array}$ & $\begin{array}{c}\text { Surface } \\
\text { roughness }\end{array}$ & MRR \\
\hline 1 & 0.543602 & 0.901247 & 0.081542 & & 0.650042 & 1 & 0 \\
2 & 0.148843 & 0.39616 & 0.189218 & & 0.974782 & 0.39299 & 0.20025 \\
3 & 0.168963 & 0.006058 & 0 & & 1 & 0.012037 & 0.185337 \\
4 & 0.129306 & 0.371799 & 0.755227 & & 0.524447 & 0.363027 & 0.713947 \\
5 & 0 & 0 & 0.822116 & & 0.159105 & 0 & 0.731856 \\
6 & 0.521785 & 0.891778 & 0.746666 & & 0.819905 & 0.993454 & 0.670744 \\
7 & 0.7121 & 1 & 1 & & 0.961398 & 0.004036 & 1 \\
8 & 1 & 0.870742 & 0.93958 & & 0 & 0.933709 & 0.9085 \\
9 & 0.533321 & 0.352234 & 0.975205 & & 0.922745 & 0.340096 & 0.934964 \\
\hline
\end{tabular}

Table 16

Normalized $\mathrm{S} / \mathrm{N}$ data-chromium alloy, stainless, and nickel steel data.

\begin{tabular}{|c|c|c|c|c|c|c|c|c|c|}
\hline & \multicolumn{3}{|c|}{ Chromium alloy steel } & \multicolumn{3}{|c|}{ Stainless steel } & \multicolumn{3}{|c|}{ Nickel steel } \\
\hline & $\begin{array}{l}\text { Precision } \\
\text { error }\end{array}$ & $\begin{array}{l}\text { Surface } \\
\text { roughness }\end{array}$ & MRR & $\begin{array}{c}\text { Precision } \\
\text { error }\end{array}$ & $\begin{array}{c}\text { Surface } \\
\text { roughness }\end{array}$ & MRR & $\begin{array}{c}\text { Precision } \\
\text { error }\end{array}$ & $\begin{array}{c}\text { Surface } \\
\text { roughness }\end{array}$ & MRR \\
\hline 1 & 0.357755 & 0.960103 & 0 & 0.393946 & 0.989939 & 0 & 0.329007 & 0.996548 & 0.037586 \\
\hline 2 & 1 & 0.403133 & 0.213114 & 0.184066 & 0.387998 & 0.058099 & 0.564503 & 0.339486 & 0 \\
\hline 3 & 0.454173 & 0.024243 & 0.301475 & 0.160212 & 0.013097 & 0.331543 & 0.739286 & 0.022063 & 0.232827 \\
\hline 4 & 0.989267 & 0.381291 & 0.754727 & 0.127066 & 0.379 & 0.800713 & 0.616 & 0.377327 & 0.723098 \\
\hline 5 & 0.337763 & 0.024581 & 0.796139 & 0 & 0 & 0.811639 & 1 & 0.005355 & 0.746097 \\
\hline 6 & 0.396324 & 0.99274 & 0.717683 & 0.401777 & 0.991944 & 0.770208 & 0.286813 & 1 & 0.677586 \\
\hline 7 & 0.798706 & 0 & 1 & 0.019355 & 1 & 1 & 0.389471 & 0 & 1 \\
\hline 8 & 0 & 1 & 0.935334 & 1 & 0.005566 & 0.947717 & 0 & 0.938058 & 0.90361 \\
\hline 9 & 0.267933 & 0.426152 & 0.958588 & 0.313833 & 0.421639 & 0.965899 & 0.287214 & 0.344941 & 0.949122 \\
\hline
\end{tabular}

Table 17

GRG-medium-carbon and low-carbon steels.

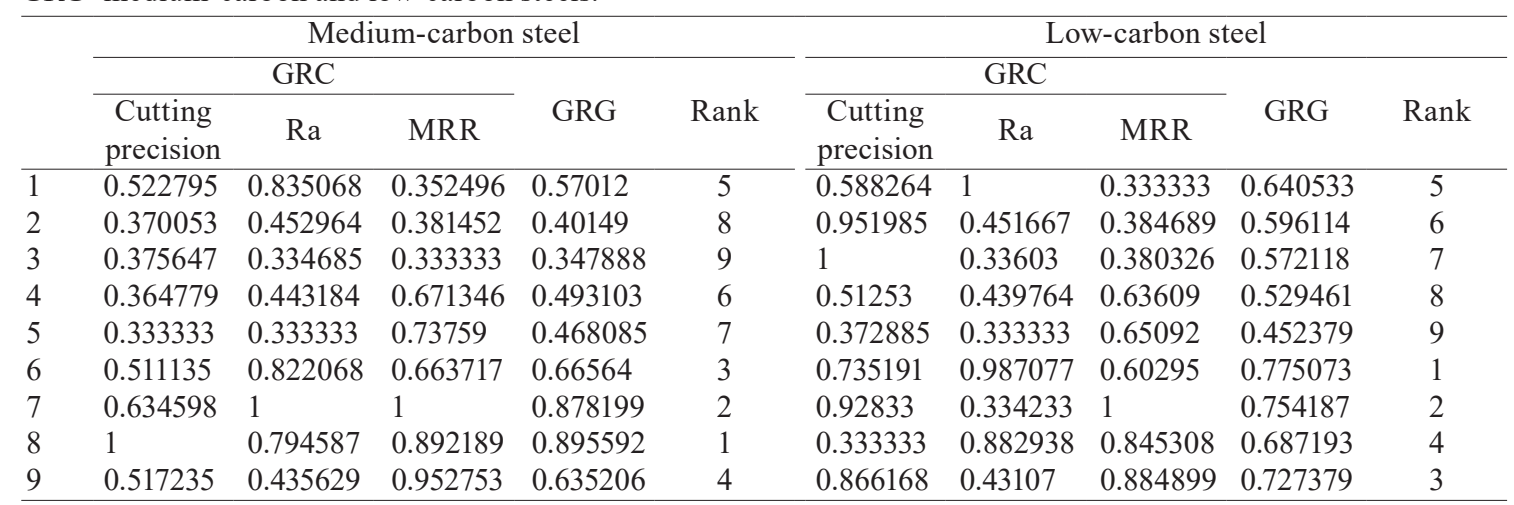


Table 18

GRG-chromium alloy, stainless, and nickel steels.

\begin{tabular}{|c|c|c|c|c|c|c|c|c|c|c|}
\hline & \multicolumn{5}{|c|}{ Chromium alloy steel } & \multicolumn{5}{|c|}{ Stainless steel } \\
\hline & \multicolumn{3}{|c|}{ GRC } & \multirow[b]{2}{*}{ GRG } & \multirow[b]{2}{*}{ Rank } & \multicolumn{3}{|c|}{ GRC } & \multirow[b]{2}{*}{ GRG } & \multirow[b]{2}{*}{ Rank } \\
\hline & $\begin{array}{l}\text { Cutting } \\
\text { precision }\end{array}$ & $\mathrm{Ra}$ & MRR & & & $\begin{array}{l}\text { Cutting } \\
\text { precision }\end{array}$ & $\mathrm{Ra}$ & MRR & & \\
\hline 1 & 0.437735 & 0.926103 & 0.333333 & 0.565723 & 7 & 0.452058 & 0.980275 & 0.333333 & 0.588555 & 5 \\
\hline 2 & 1 & 0.455844 & 0.388535 & 0.614793 & 5 & 0.379958 & 0.449639 & 0.346764 & 0.392121 & 8 \\
\hline 3 & 0.478091 & 0.338809 & 0.417179 & 0.41136 & 9 & 0.373193 & 0.33627 & 0.427915 & 0.379126 & 9 \\
\hline 4 & 0.978985 & 0.446944 & 0.670895 & 0.698941 & 2 & 0.364184 & 0.44603 & 0.715014 & 0.508409 & 6 \\
\hline 5 & 0.430205 & 0.338887 & 0.710367 & 0.493153 & 8 & 0.333333 & 0.333333 & 0.726364 & 0.464343 & 7 \\
\hline 6 & 0.453032 & 0.985688 & 0.639127 & 0.692615 & 3 & 0.455281 & 0.984143 & 0.685127 & 0.708184 & 3 \\
\hline 7 & 0.712968 & 0.333333 & 1 & 0.6821 & 4 & 0.337691 & 1 & 1 & 0.77923 & 1 \\
\hline 8 & 0.333333 & 1 & 0.885479 & 0.739604 & 1 & 1 & 0.334575 & 0.905332 & 0.746636 & 2 \\
\hline \multirow[t]{3}{*}{9} & 0.405822 & 0.465615 & 0.923511 & 0.598316 & 6 & 0.421526 & 0.463667 & 0.936153 & 0.607115 & 4 \\
\hline & \multicolumn{5}{|c|}{ Nickel steel } & & & & & \\
\hline & \multicolumn{3}{|c|}{ GRC } & \multirow[b]{2}{*}{ GRG } & \multirow[b]{2}{*}{ Rank } & & & & & \\
\hline & $\begin{array}{l}\text { Cutting } \\
\text { precision }\end{array}$ & $\mathrm{Ra}$ & MRR & & & & & & & \\
\hline 1 & 0.426988 & 0.993142 & 0.3419 & 0.587344 & 5 & & & & & \\
\hline 2 & 0.534475 & 0.430843 & 0.333333 & 0.432884 & 9 & & & & & \\
\hline 3 & 0.657278 & 0.338309 & 0.394579 & 0.463389 & 8 & & & & & \\
\hline 4 & 0.565611 & 0.445366 & 0.643581 & 0.551519 & 7 & & & & & \\
\hline 5 & 1 & 0.334528 & 0.663216 & 0.665914 & 3 & & & & & \\
\hline 6 & 0.412138 & 1 & 0.607966 & 0.673368 & 2 & & & & & \\
\hline 7 & 0.450236 & 0.333333 & 1 & 0.594523 & 4 & & & & & \\
\hline 8 & 0.333333 & 0.889772 & 0.838378 & 0.687161 & 1 & & & & & \\
\hline 9 & 0.412274 & 0.432878 & 0.907642 & 0.584265 & 6 & & & & & \\
\hline
\end{tabular}

The Taguchi quality experimental method was used to find a single optimization target combination relative to the previous combination. Thus, with the assistance of GRA, the machine users can select a "single target" or search for "optimal parameters for multiple optimizations".

\section{Conclusion}

We presented the results of an investigation of optimal parameters for the lathe machining of several different materials using the Taguchi method and GRA. The experimental results show the impacts of the cutting precision targets of various materials, with cutting depth and spindle speed having the greatest impacts. Spindle speed affected surface roughness, and cutting depth affected the material removal rate. These factors can be used by machine operators to adjust and improve target selection. The optimal parameter combinations found in this study reduced quality loss more significantly than previous combinations. This achieved the purpose of the search for optimized parameters. In the future, other directions, such as the analysis of variance (ANOVA) to derive more specific selections and enhance parameter optimization, could be investigated. 


\section{Acknowledgments}

The authors acknowledge with gratitude a funding grant from the Ministry of Science and Technology for the project "Domestic Lathe Machine Tool Spindle Peripheral Smart Functional Technology Development" (project No. MOST 108-2218-E-167-001). This work was also financially supported by the Teacher Education Research Project of Qujing Normal University under Grant No. 2019JZ001.

\section{References}

1 K. Venkatesh, M. C. Zhou, and R. J. Caudill: J. Intell. Manuf. 8 (1997) 215. https://oi.org/10.1023/ A:1018573224739

2 D. E. Dimla: Int. J. Mach. Tools Manuf. 40 (2000) 1073. https://doi.org/10.1016/s0890-6955(99)00122-4

3 A. Siddhpura and R. Paurobally: Int. J. Adv. Manuf. Technol. 65 (2013) 371. https://doi.org/10.1007/s00170012-4177-1

4 D. Choi, W. T. Kwon, and C. N. Chu: Int. J. Adv. Manuf. Technol. 15 (1999) 305. https://doi.org/10.1007/ s001700050071

5 D. Yan, T. I. El-Wardany, and M. A. Elbestawi: Int. J. Mach. Tools Manuf. 35 (1995) 383. https:// doi. org/10.1016/0890-6955(94)e0021-a

6 M. Nouri, B. K. Fussell, B. L. Ziniti, and E. Linder: Int. J. Mach. Tools Manuf. 89 (2015) 1. https:// doi. org/10.1016/j.ijmachtools.2014.10.011

7 R. K. Bharilya, R. Malgaya, L. Patidar, R. K. Gurjar, and A. K. Jha: Mater. Today: Proc. 2 (2015) 2300. https:// doi.org/10.1016/j.matpr.2015.07.268

8 C. Pislaru, J. M. Freeman, and D. G. Ford: Int. J. Mach. Tools Manuf. 43 (2003) 987. https:// doi.org/10.1016/ S0890-6955(03)00104-4

9 W. Rmili, A. Ouahabi, R. Serra, and R. Leroy: Measurement 77 (2016) 117. https:// doi.org/10.1016/ j.measurement.2015.09.010

10 B. Cuka and D. W. Kim: Rob. Comput. Integr. Manuf. 47 (2017) 22. https://doi.org/10.1016/j.rcim.2016.12.009

11 J. S. Pang, M. N. M. Ansari, O. S. Zaroog, M. H. Ali, and S. M. Sapuan: HBRC J. 10 (2014) 138. https://doi. org/10.1016/j.hbrcj.2013.09.007

12 B. Das, S. Roy, R. N. Rai, and S. C. Saha: Eng. Sci. Technol. 19 (2016) 857. https://doi.org/10.1016/ j.jestch.2015.12.002

13 B. Das, S. Roy, R. N. Rai, and S. C. Saha: Eng. Sci. Technol. 19 (2016) 279. https://doi.org/10.1016/ j.jestch.2015.08.002

14 A. Gupta, H. Singh, and A. Aggarwal: Expert Syst. Appl. 38 (2011) 6822. https://doi.org/10.1016/ j.eswa.2010.12.057

15 I. Asiltürk, S. Neseli, and M. A. Ince: Measurement 78 (2016) 120. https://doi.org/10.1016/ j.measurement.2015.09.052

16 C. B. Li, Q. G. Xiao, Y. Tang, and L. Li: J. Clean Prod. 135 (2016) 263. https://doi.org/10.1016/j.jclepro.2016.06.097

17 S. Ajith Arul Daniel, R. Pugazhenthi, R. Kumar, and S. Vijayananth: Def. Technol. 15 (2019) 545. https://doi. org/10.1016/j.dt.2019.01.001

18 T. Thankachan, K. S. Prakash, R. Malini, S. Ramu, P. Sundararaj, S. Rajandran, D. Rammasamy, and S. Jothi: Appl. Surf. Sci. 472 (2019) 22. https://doi.org/10.1016/j.apsusc.2018.06.117

19 N. Tamiloli, J. Venkatesan, and B. V. Ramnath: Measurement 84 (2016) 68. https://doi.org/10.1016/ j.measurement.2016.02.008

20 R. L. Edgeman: Technometrics 34 (1992) 230. https://dx.doi.org/10.1080/00401706.1992.10484920

21 F. C. Wu: Int. J. Adv. Manuf. Technol. 20 (2002) 749. https://doi.org/10.1007/s001700200233

22 J. W. K. Chan and T. K. L. Tong: Mater. Des. 28 (2007) 1539. https:/doi.org/10.1016/j.matdes.2006.02.016

23 Y. Shrivastava and B. Singh: Eur. J. Mech. A Solids 70 (2018) 238. https://doi.org/10.1016/ j.euromechsol.2018.03.009 


\section{About the Authors}

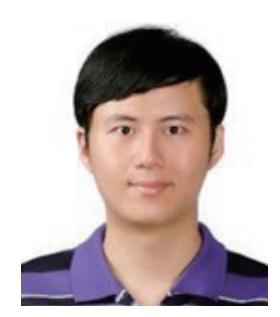

Bo-Lin Jian received his B.S. degree from the Department of Electrical Engineering, National Formosa University in 2009 and his degree M.S. in materials science and engineering from the National Taiwan University of Science and Technology in 2011. His Ph.D. degree was awarded by the Department of Aeronautics and Astronautics, National Cheng Kung University in 2017. He is an assistant professor in the Department of Electrical Engineering, National Chin-Yi University of Technology, Taichung, Taiwan. His current research interests include signal and image processing, machine learning, and control systems. (BoLin@ncut.edu.tw)

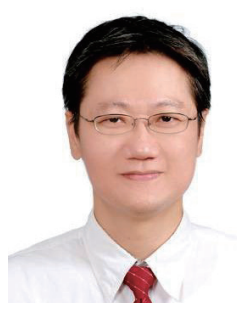

Cheng-Chi Wang serves as a distinguished professor and also the dean of the Graduate Institute of Precision Manufacturing in National Chin-Yi University of Technology in Taiwan. His current research involves intelligent machining and manufacturing, nonlinear dynamic analysis, simulation and optimization, air lubrication system and signal processing. (wcc@ncut.edu.tw)

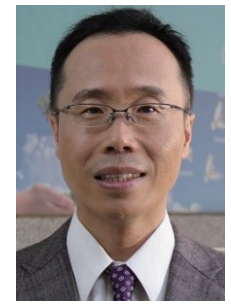

Her-Terng Yau received his B.S. degree from National Chung Hsing University, Taichung, Taiwan, in 1994 and his M.S. and Ph.D. degrees from National Cheng Kung University, Tainan, Taiwan, in 1996 and 2000, respectively, all in mechanical engineering. He is currently a professor in the Department of Electrical Engineering, National Chin-Yi University of Technology, Taichung, Taiwan. His research interests include energy converter control, system control of mechatronics, nonlinear system analysis, and control. He is the author of more than 150 research articles on a wide variety of topics in mechanical and electrical engineering.

(htyau@ncut.edu.tw)

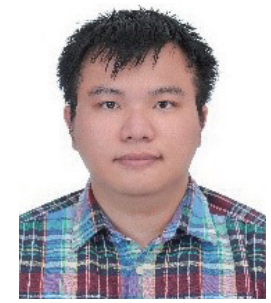

Li-Wei Wu received his B.S. and M.S. degrees from the Department of Electrical Engineering, National Chin-Yi University of Technology in 2017 and 2019, respectively. (ff9456gdgs@gmail.com)

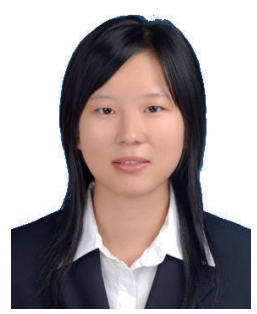

An-Hong Tian received her B.S. and M.S. degrees from Chongqing University of Posts and Telecommunications, China, in 2007 and 2010, respectively. From 2011 to 2016, she was a lecturer at Qujing Normal University, China. Since 2017, she has been an associate professor at Qujing Normal University. Her research interests are in remote sensing, wireless navigation, and artificial intelligence. (tianah@mail.qjnu.edu.cn) 